\title{
Pratiques
}

Linguistique, littérature, didactique

183-184| 2019

oralité, littératie

\section{Langue écrite}

\section{Pierre-Yves Testenoire}

\section{(2) OpenEdition}

\section{Journals}

Édition électronique

URL : http://journals.openedition.org/pratiques/6757

DOI : $10.4000 /$ pratiques. 6757

ISSN : 2425-2042

\section{Éditeur}

Centre de recherche sur les médiations (CREM)

Référence électronique

Pierre-Yves Testenoire, «Langue écrite », Pratiques [En ligne], 183-184 | 2019, mis en ligne le 30 décembre 2019, consulté le 09 octobre 2020. URL : http://journals.openedition.org/pratiques/6757 : DOI : https://doi.org/10.4000/pratiques.6757

Ce document a été généré automatiquement le 9 octobre 2020.

(c) Tous droits réservés 


\title{
Langue écrite
}

\author{
Pierre-Yves Testenoire
}

1 Les problèmes soulevés par la notion de "langue écrite " sont nombreux car, comme l'écrivait Nina Catach (1988: 17), elle est «un concept par essence polysémique ». L'expression est employée de façon récurrente dans le discours métalinguistique sans être nécessairement conceptualisée. Si l'on écrit beaucoup sur la langue écrite - ses propriétés, ses rapports avec la langue parlée, variables selon les idiomes, son rôle en synchronie comme en diachronie - on se risque rarement à la définir. Et lorsque l'on fait, les définitions vont de propositions a minima, «la langue sous forme écrite », par exemple, chez Benveniste, à des essais plus ambitieux mais contradictoires entre eux : « langue plus réfléchie, plus logique que la langue parlée » (Bally), « expression la plus caractéristique des langues communes » (Vendryes), «système de moyens graphiques reconnu comme norme au sein d'une communauté » (Vachek, 1939)...

2 Ces différentes acceptions proviennent de la diversité des options sur la question, ancienne et débattue, du rapport entre langue et écriture. Selon que l'on identifie la langue à sa manifestation orale ou que l'on considère l'écrit et l'oral comme deux systèmes distincts ayant leurs spécificités, l'acception du syntagme "langue écrite " change du tout au tout. La première position, que l'on qualifie classiquement de phonocentriste, fait de la langue une forme dont la substance, orale ou écrite, est indifférente. La langue écrite ne désigne alors pas une langue à proprement parler (un système sémiologique propre), mais elle désigne une variation au sein d'une même langue en termes - selon les cas - de substance, de comportement communicatif, de norme, de registre, de style... La seconde position fait, au contraire, de la langue écrite un système distinct de la langue parlée d'un même idiome, auquel elle reconnaît une autonomie. Langue écrite et langue parlée forment alors bien deux systèmes sémiologiques, deux langues, qui peuvent interférer mais ne sont pas en relation de dépendance. Cette partition, très schématique, entre courant phonocentriste et courant autonomiste dessine deux acceptions incompatibles de la notion de langue écrite ; elle croise la partition entre représentations continuiste et discontinuiste de la dualité oral/ écrit qui traverse la linguistique moderne. Les linguistes $\mathrm{du} \mathrm{xx}^{\mathrm{e}}$ siècle qui ont théorisé le rapport entre langue et écriture peuvent être distribués entre ces deux courants ${ }^{1}$. A 
la première tradition, majoritaire, on rattache : le Saussure du CLG, Sapir, Bloomfield, Jakobson, Benveniste, Martinet, Gak, Catach, Koch \& Oesterreicher (avec des nuances importantes entre eux); à la seconde, qui confère à la langue écrite le sens plein de langue : Hjelmslev, Uldall, Vachek, Pulgram, Harris, Anis (là encore, avec d'importantes nuances).

3 Ce qui est remarquable, mais a déjà été relevé avant nous, c'est que ces deux traditions se réfèrent toutes les deux à Saussure, découlant de deux lectures structurales concurrentes du CLG (v. Arrivé 1983, Chiss \& Puech 1983, Anis et al. 1988). Si les paradoxes de la position saussurienne sur l'écriture autorisent aussi bien une approche autonomiste que phonocentriste (Testenoire 2017), la notion de langue écrite n'est pas chez lui - pas plus que chez ses contemporains - véritablement conceptualisée. La plupart des linguistiques de la charnière des $\mathrm{XIX}^{\mathrm{e}}$ et $\mathrm{xX}^{\mathrm{e}}$ siècles réfléchissent à ce qui différencie la langue parlée de la langue écrite (v. Testenoire 2016; Combettes 2018), mais ils définissent rarement ce qu'ils entendent par cette dernière. L'expression est, en outre, concurrencée par la notion contiguë de langue littéraire, prise alors majoritairement dans son sens étymologique, c'est-à-dire au sens de «langue de l'écrit ", ou par extension de "langue commune » ou de "langue officielle ", et moins souvent au sens de «langue de la littérature $»^{2}$.

Dans les ouvrages de linguistique de langue française de cette période (Bréal, Paris, Henry, Saussure, Brunot, Meillet, Bally, Sechehaye, Vendryes, Dauzat), nous avons pu identifier trois métaphores pour dire la relation de l'écriture à la langue, et par-là pour représenter la langue écrite :

- Une métaphore organiciste : Les productions écrites sont alors comparées à des fossiles, des coquilles, des éléments ossifiés ou encore à des végétaux mis dans un herbier. Selon la métaphore de «la vie du langage » particulièrement productive à l'époque (Klippi 2010), la langue parlée s'oppose à la langue écrite comme l'eau vive s'oppose à la glace ou à des flaques immobiles et stagnantes.

- Une métaphore textile : L'écriture, perçue comme un obstacle pour l'observation de la langue, est comparée à une enveloppe, à un voile ou à toute sorte de vêtements - manteau, camisole, voire guenille - jetés sur le corps de la langue.

- Une métaphore mimétique : L'écriture est assimilée à une image, un portrait ou une photographie de la langue. L'analyse critique de l'écriture comme représentation trompeuse s'inscrit dans la droite ligne de la critique platonicienne de la mimésis. (Testenoire 2016)

La linguistique du début $\mathrm{du} \mathrm{xx}^{\mathrm{e}}$ siècle cherche à s'autonomiser : en réaction contre le primat de la philologie et contre les approches puristes de la langue, elle tend à situer son véritable objet dans la langue parlée. La langue écrite est alors principalement décrite en négatif. On la dote de traits variables en opposition à ce qui serait l'objet réel de la linguistique. Langue écrite prend les sens de "langue mise par écrit » mais aussi de "langue commune », de "norme élevée », de «langue intellectuelle »... L'essai de Vachek (1939) qui propose une réflexion en positif de la notion de langue écrite constitue, de ce point de vue, une exception. Et c'est à partir des années 1960, avec le développement de la sociolinguistique et des recherches sur l'oral, que l'on prend progressivement conscience du cumul des oppositions - médiales (acoustique/ graphique), variationnelles (norme basse/norme élevée), communicatives (proximité/ éloignement) - dont les expressions polysémiques de "langue parlée » et de "langue écrite » sont devenues, au cours du siècle, les réceptacles. 


\section{BIBLIOGRAPHIE}

ARRIVÉ, M. (1983). « Les danois aux prises avec la substance de l'encre », Langue française 59, p. 25-30.

ANIS, J. (1988). L'écriture. Théories et descriptions. Paris/Bruxelles : Éditions universitaires/De Boeck. ANIS, J. (2000), « Vers une sémiolinguistique de l'écrit ». Linx. Revue des linguistes de l'Université Paris Ouest Nanterre La Défense 43, p. 29-44. DOI : https://doi.org/10.4000/linx.1046.

BLANCHE-BENVENiste, C. \& JEANJEAN, C. (1987), Le français parlé. Transcription et édition. Paris : Didier.

BÉGUELIN, M.-J. (1998), « Le rapport écrit-oral. Tendances dissimilatrices, tendances

assimilatrices », Cahiers de linguistique française 20, p. 229-253.

CATACH, N. (éd.) (1988). Pour une théorie de la langue écrite. Paris : CNRS.

COMBETTES, B. (2017), « L'opposition langue parlée/langue écrite dans la linguistique historique de tradition française (1860-1930) », Langages 208, p. 69-82. DOI : https://doi.org/10.3917/lang. 208.0069

CHISS, J.-L. \& PUECH, C. (1983), « La linguistique et la question de l'écriture : enjeux et débats autour de Saussure et des problématiques structurales ", Langue française 59, p. 5-24.

KLIPPI, C. (2010), La vie du langage. La linguistique dynamique en France de 1864 à 1916, Lyon, ENS éditions.

KOCH, P. \& OESTERREICHER, W. (2001) « Langage oral et langage écrit ». In : Holtus, G., Metzeltin, M., \& Schmitt, C. (éds), Lexikon der romanistischen Linguistik. Band I, 2: Methodologie. Tubingue : Max Niemeyer Verlag, p. 584-627.

MARHER, R. (2017). Phonographie. La représentation écrite de l'oral en français. Berlin : De Gruyter.

PHILIPPE, G. \& PIAT, J. (éds.) (2009). La langue littéraire. Une histoire de la prose en France de Gustave Flaubert à Claude Simon. Paris : Fayard.

TESTENOIRE, P.-Y. (2016). « Sur la conceptualisation de la langue écrite dans les théorisations linguistiques du début du XX ${ }^{\mathrm{e}}$ siècle ». Les dossiers d'HEL 9, p. 34-46. En ligne : http:// htl.linguist.univ-paris-diderot.fr/hel/dossiers/numero9.

TESTENOIRE, P.-Y. (2017). « "Le langage est une institution SANS ANALOGUE (si l'on y joint l'écriture)" : L'écriture comme problèmes dans la réflexion théorique de Saussure ». Semiotica 217, p. 117-133. DOI : https://doi.org/10.1515/sem-2016-0061.

VACHEK, J. (1939), « Zum Problem der geschriebenen Sprache ». Travaux du cercle linguistique de Prague 8, p. 94-104.

\section{NOTES}

1. Nous laissons de côté les autres approches que la question de l'écriture a suscité au $\mathrm{xx}^{\mathrm{e}}$ siècle: historique (Février, Gelb), philosophique (Derrida), anthropologique (Calame-Griaule, Goody), sémiotique (Christin), cognitives... 
2. Cette dernière acception se rencontre cependant s'impose progressivement dans le premier tiers du $\mathrm{xx}^{\mathrm{e}}$ siècle (Philippe \& Piat, 2009). 